\title{
A Spectral Theory for the Reduced Wave Equation with a Complex Refractive Index
}

\author{
By
}

Teruo IKEBE*

\section{§1. Introduction}

The present article develops a spectral theory (with main emphasis on the continuous spectrum) for the reduced wave equation with a complex refractive index $n(x)$

$$
\Delta u+\kappa^{2} n(x)^{2} u=0
$$

over all of $\mathbf{R}^{3}$, the Euclidean 3 -space, where $\kappa$ is the complex frequency parameter. The $n(x)$ is assumed throughout to be a bounded (measurable) function such that

$\operatorname{Re} n(x) \geqq 1, \operatorname{Im} n(x) \geqq 0, \operatorname{Re} n(x) \geqq \operatorname{Im} n(x)$, and $n(x)-1$ has $a$ compact support.

In connection with equation $(*)$ the operator $-n^{-2} \Delta$ will be considered in the Hilbert space $\mathbf{H}=L_{2}\left(\mathbf{R}^{3}\right)$, square integrable functions over $\mathbb{R}^{3}$, whose norm and inner product will be denoted by \|\| and $($,$) . More$ explicitly, let $H_{0}$ be the unique self-adjoint realization of $-\Delta$ in $\mathbf{H}$ and $N$ the bounded operator of multiplication by $n(x)^{-2}$, and consider the unambiguous product $H=N H_{0}$ with domain identical with that of $H_{0}$ : $D(H)=D\left(H_{0}\right)$, the latter consisting, as is well known, of $f \in \mathbb{H}$ with second-order $L_{2}$ derivatives. Together with $H$ it is convenient to consider its adjoint $H^{*}=H_{0} N^{*}$ with $D\left(H^{*}\right)=N^{*-1} D\left(H_{0}\right)$.

Additive non-self-adjoint perturbations of $H_{0}$ have been investigated,

Received September 6, 1972.

Communicated by S. Matsuura.

* Department of Mathematics, Faculty of Science, Kyoto University, Kyoto, Japan. 
for example, by Mochizuki [1] and Goldstein [2] (see also papers cited in [1] and [2], and "Problemy Matematičeskoì Fiziki", Leningrad Univ. (ed. by M.Š. Birman)). ${ }^{1)}$ Here, a simple example of multiplicative perturbation of $H_{0}$ will be dealt with by the so-called integral equation method which has been employed by Mochizuki [1] and by Povzner [3] and Ikebe $[4]$ in the self-adjoint case. The results obtained here will possess much similarity with those of $[1],[2],[3]$ and $[4]$, and, therefore, details of the argument will very often be left out, and just how to proceed will be indicated.

The contents of the present paper will be outlined. Section 2, starting with resolvent equations relating $H$ and $H^{*}$ with $H_{0}$, introduces some auxiliary compact operators, the compactness being useful in exposing the behavior of the resolvents of $H$ and $H^{*}$ in a neighborhood of the continuous spectrum (this sort of technique is usually called the limiting absorption method), and reveals a very rough structure of the spectrum of $H$ and $H^{*}$; for instance, the essential spectrum of $H$ and $H^{*}$ is identical with that of $H_{0}$. In section 3 certain classes of generalized eigenfunctions for $H$ and $H^{*}$ are introduced as well as off-real distorted plane waves, the former being determined as boundary values of the latter (which is nothing but a limiting absorption technique). Sections 4 and 5 are of a preparatory character, where following the idea of Shenk [5] a convenient representation of the resolvent involving the off-real distorted plane waves is given, and spectral measures $E(\Delta)$ and $E^{*}(\Delta)$ associated with $H$ and $H^{*}$ are defined for appropriate Borel sets $\Delta$ of $[0, \infty)$ (bounded away from a certain discrete set of exceptional points). In the final section 6 is expounded a spectral representation for an absolutely continuous part of $H, H(\Delta)=E(\Delta) H$, in terms of generalized Fourier transforms that give rise to a unitary map from $E(\Delta) \mathbf{H}$ onto an $L_{2}$ space over a domain of $\mathbf{R}^{3}$ determined by $\Delta:$ For $\xi \in \mathbf{R}^{3}$ let $\varphi(x, \xi)$ and $\varphi^{*}(x, \xi)$ be generalized

1) In [1] has been studied the operator $-\Delta+q(x)$ in $\mathbf{R}^{3}$, where the complex potential $q(x)$ has been assumed to behave like $|x|^{-2-\varepsilon}, \varepsilon>0$, at infinity. Some results of [1] have recently been extended by Saito [11] to the case where $q(x)=$ $O\left(|x|^{-1-\varepsilon}\right), \varepsilon>0$. Second-order perturbations of $-\Delta$, of which the present problem is a special case, have been treated in [2] from the additive perturbation view-point. 
eigenfunctions with generalized eigenvalue $|\xi|^{2}$ for $H$ and $H^{*}$. Then the generalized Fourier transform is given by

$$
\hat{f}^{*}(\xi)=(2 \pi)^{-3 / 2} \int \overline{\varphi^{*}(x, \xi)} f(x) d x
$$

and $H f$ turns through this transformation into $(H f)^{\wedge}(\xi)=|\xi|^{2} \hat{f}^{*}(\xi)$, provided $|\xi|^{2}$ lies in a suitable $\Delta$. The original $f$ may be reinstated by an inversion formula like (but not precisely the same as)

$$
f(x)=(2 \pi)^{-3 / 2} \int \varphi(x, \xi) \hat{f}^{*}(\xi) d \xi
$$

This situation corresponds exactly to the spectral representation for $E_{0}(\Delta) H_{0}$, where $E_{0}$ is the spectral measure for $H_{0}$, in terms of the ordinary Fourier transforms.

Now several comments and remarks are due.

No restriction other than boundedness has been placed upon the regularity of the refractive index. Consequently, the entire results here are applicable to the interface or transmission problem (Beugungsproblem) in which the refractive index suffers a discontinuity across an interface dividing different media.

The compactness of the support of the function $n(x)-1$ simplifies the discussion in many respects. It would be possible to go without this compactness assumption, though various technical alterations would have to be introduced depending on various diminishing orders of $n(x)-1$ at infinity.

The higher-dimensional analogue of the problem treated here seems to produce no essential difficulties. The operator $Q(\kappa)$ introduced in the next section may not be expected to be of Hilbert-Schmidt type as in the three-dimensional case. The trouble would be overcome by retaining the (non-Hilbert-Schmidt) compactness of $Q(\kappa)$, however.

Avila and Haggerty [6] have reduced a sort of multiplicative perturbation problem to an additive one for the acoustic equation with a variable density. Such a device does not seem successful as things stand in the present case. And no attempts are made here toward this kind of 
approach.

\section{§2. Resolvent Equations, Eigenvalues and Exceptional Points}

The resolvents $R_{0}(z)=R\left(z: H_{0}\right)$ and $R(z)=R(z: H)$ are by definition the bounded operators $\left(H_{0}-z\right)^{-1}$ and $(H-z)^{-1}$ whenever they exist, i.e., when $z$ is in their respective resolvent sets $\rho\left(H_{0}\right)$ and $\rho(H)$. The (second) resolvent equation interconnecting $R_{0}(z)$ and $R(z)$ is the starting point of the whole development that follows, and is most easily obtained by looking at the equation

$$
(H-z) R(z) f=f \text { or } N H_{0} R(z) f-z R(z) f=f .
$$

The resulting resolvent equation reads as follows:

$$
R(z)-R_{0}(z) N^{-1}=z R_{0}(z) N^{-1}(1-N) R(z) \quad \text { for } z \in \rho\left(H_{0}\right) \cap \rho(H) .
$$

Here it is of course assumed that $\rho\left(H_{0}\right) \cap \rho(H)$ is not empty. Similarly, one can obtain for $R^{*}(z)=R\left(z: H^{*}\right)$

$$
\begin{array}{r}
R^{*}(z)-N^{*^{-1}} R_{0}(z)=z N^{*^{-1}} R_{0}(z)\left(1-N^{*}\right) R^{*}(z) \\
\qquad \text { for } z \in \rho\left(H_{0}\right) \cap \rho\left(H^{*}\right) .
\end{array}
$$

Observing the easily obtainable relation $R^{*}(\bar{z})=R(z)^{*}$ gives in virtue of (2.1) and (2.2)

$$
R(z)-R_{0}(z) N^{-1}=z R(z)(1-N) R_{0}(z) N^{-1} \text { for } z \in \rho\left(H_{0}\right) \cap \rho(H)
$$

and

$$
\begin{array}{r}
R^{*}(z)-N^{*^{-1}} R_{0}(z)=z R^{*}(z)\left(1-N^{*}\right) N^{*^{-1}} R_{0}(z) \\
\text { for } z \in \rho\left(H_{0}\right) \cap \rho\left(H^{*}\right) .
\end{array}
$$

Equations (2.1)-(2.4) are the desired resolvent equations. ${ }^{2)}$ Introducing multiplicative operators $A$ and $B$ by

2) No use of equations (2.3) and (2.4) will be made in subsequent discussions, however. 


$$
(A f)(x)=\left|n(x)^{2}-1\right|^{1 / 2} f(x),(B f)(x)=\operatorname{sgn}\left(n(x)^{2}-1\right) A(x) f(x)
$$

so that $N^{-1}(1-N)=B A=A B$ and $1-N^{*}=A N^{*} B^{*}$, one obtains in view of (2.1) and (2.2)

$$
\begin{gathered}
A R(z)=A R_{0}(z) N^{-1}+z A R_{0}(z) B A R(z), \\
N^{*} B^{*} R^{*}(z)=B^{*} R_{0}(z)+z B^{*} R_{0}(z) A N^{*} B^{*} R^{*}(z),
\end{gathered}
$$

where $\operatorname{sgn} a=\frac{a}{|a|}$ if $a \neq 0$ and $=0$ if $a=0$. In order to investigate equations (2.5) and $(2.5)^{*}$ one introduces

$$
Q(\kappa)=\kappa^{2} A R_{0}\left(\kappa^{2}\right) B \text { and } Q^{*}(\kappa)=\kappa^{2} B^{*} R_{0}\left(\kappa^{2}\right) A=Q(-\bar{\kappa})^{*}
$$

at first for $\kappa \in \mathbb{C}$ with $\operatorname{Im} \kappa>0$. Obviously $Q(\kappa)\left[Q^{*}(\kappa)\right]$ is an integral operator with kernel

$$
\frac{\kappa^{2}}{4 \pi} A(x) \frac{\mathrm{e}^{\mathrm{i} \kappa|x-y|}}{|x-y|} B(y) \quad\left[\frac{\kappa^{2}}{4 \pi} B^{*}(x) \frac{\mathrm{e}^{\mathrm{i} \kappa \mid x-y !}}{|x-y|} A(y)\right] .
$$

With this explicit form of the kernel the operator-valued function $Q(\kappa)$ $\left[Q^{*}(\kappa)\right]$ can be extended to the entire $\kappa$-plane $\mathbf{C}$ and the following lemma may be proved without difficulty once the compactness of the supports of $A(x)$ and $B(x)$ is noted.

Lemma 2.1. $Q(\kappa)\left[Q^{*}(\kappa)\right], \kappa \in \mathbf{C}$, is a compact linear operator on $\mathbf{H}$ of Hilbert-Schmidt type, and is an entire holomorphic function of $\kappa$.

In passing it should be noted that when (2.5) $\left[(2.5)^{*}\right]$ is viewed as an equation for the unknown $A R(z)\left[N^{*} B^{*} R^{*}(z)\right]$, it is uniquely solvable if and only if $1-Q(\sqrt{z})\left[1-Q^{*}(\sqrt{z})\right]$ is invertible, where and hereafter by $\sqrt{z}$ is meant that branch of the square root of $z$ which has positive imaginary part. And in this case $R(z)\left[R^{*}(z)\right]$ can be expressed as

$$
\begin{gathered}
R(z)=R_{0}(z) N^{-1}+z R_{0}(z) B(1-Q(\sqrt{z}))^{-1} A R_{0}(z) N^{-1} \\
{\left[R^{*}(z)=N^{*^{-1}} R_{0}(z)+z N^{*^{-1}} R_{0}(z) A\left(1-Q^{*}(\sqrt{z})\right)^{-1} B^{*} R_{0}(z)\right] .}
\end{gathered}
$$

Definition 2.2. A complex number $\kappa$ is said to be $Q \cdot\left[Q^{*_{-}}\right]$excep- 
tional if 1 is an eigenvalue of $Q(\kappa)\left[Q^{*}(\kappa)\right]$ (in which case $1-Q(\kappa)$ $\left[1-Q^{*}(\kappa)\right]$ is not invertible).

Lemma 2.3. The $Q-\left[Q^{*}-\right]$ exceptional points form a discrete set (having no finite accumulation point).

The above lemma follows directly from lemma 2.1 and a well-known theorem concerning holomorphic families of compact operators (see Kato [7], p. 370).

The following lemma gives an interrelation between the exceptional points and the eigenvalues of $H$ or $H^{*}$, and can be proved in a standard way (see, e.g., $[1]$, p. 430 ff. and [4], p. 15).

Lemma 2.4. Let $\operatorname{Im} \kappa>0$. Then $\kappa$ is $Q-\left[Q^{*_{-}}\right]$exceptional if and only if $\kappa^{2}$ is an eigenvalue of $H\left[H^{*}\right]$ of finite multiplicity.

The set of all $Q \cdot\left[Q^{*}\right]$ exceptional points will be denoted by $\tilde{\Sigma}$ $\left[\tilde{\Sigma}^{*}\right]$, while $\Sigma\left[\Sigma^{*}\right]$ will designate its intersection with the closed upper half-plane $(\operatorname{Im} \kappa \geqq 0)$. The next lemma provides a little more detailed information about the location of the exceptional points than lemmas 2.3 and 2.4, but will not be made explicit use of in the sequel. The proof is not hard, but is the only place where one has to make explicit use of the conditions $\operatorname{Im} n(x) \geqq 0, \operatorname{Re} n(x) \geqq 1$ and $\operatorname{Re} n(x) \geqq \operatorname{Im} n(x)$, and will be sketched in the appendix.

Lemma 2.5. $\left\{\kappa \mid 0 \leqq \arg \kappa \leqq \frac{\pi}{4}\right.$ or $\left.\frac{\pi}{2}<\arg \kappa \leqq \frac{3}{4} \pi\right\} \cap \Sigma=\phi$, $\left\{\kappa \mid \frac{\pi}{4} \leqq \arg \kappa<\frac{\pi}{2}\right.$ or $\left.\frac{3}{4} \pi \leqq \arg \kappa \leqq \pi\right\} \cap \Sigma^{*}=\phi . \quad$ If, in addition, the support of $\operatorname{Im} n(x)$ contains an open set, then $\left\{\kappa \mid \arg \kappa=\frac{\pi}{2}\right\}$ has no intersection with $\Sigma$ or $\Sigma^{*}$, and, in particular, there exist no negative eigenvalues of $H$ or $H^{*}$.

It is noted here that $H$ and $H^{*}$ are densely defined, closed linear operators in $\mathbf{H}$, and that the residual spectrum of a closed linear operator $H, \sigma_{\mathrm{r}}(H)$, is characterized by 


$$
z \in \sigma_{\mathrm{r}}(H) \Leftrightarrow \bar{z} \in \sigma_{\mathrm{p}}\left(H^{*}\right) \text { and } z \notin \sigma_{\mathrm{p}}(H)
$$

where $\sigma_{\mathrm{p}}(H)$ denotes the point spectrum of $H$. Consequently, by using lemma 2.4 it can be easily shown that both $\sigma_{\mathrm{r}}(H)$ and $\sigma_{\mathrm{r}}\left(H^{*}\right)$ are empty. In this case a point $\lambda \in \sigma(H)$, the spectrum of $H$, can be characterized by the existence of a normalized sequence $\left\{f_{n}\right\}$ such that

$$
(H-\lambda) f_{n} \rightarrow 0 \text { as } n \rightarrow \infty \text {. }
$$

If there exists a non-(relatively) compact such sequence, $\lambda$ is said to belong to the essential spectrum $\sigma_{\mathrm{e}}(H)$ of $H$, and otherwise, $\lambda$ is said to belong to the discrete spectrum $\sigma_{\mathrm{d}}(H)$ of $H$. Thus $\sigma(H)=\sigma_{\mathrm{d}}(H) \cup \sigma_{\mathrm{e}}(H)$ and $\sigma_{\mathbf{r}}(H)=\phi$.

In the above-mentioned case the proof of Weyl's theorem which asserts the invariance of the essential spectrum under compact perturbations in the self-adjoint case remains valid with some elementary reduction such as the one done in $[4]$, p. 19 . Thus with lemma 2.1 one can conclude that $\sigma_{\mathrm{e}}(H)=\sigma_{\mathrm{e}}\left(H^{*}\right)=\sigma_{\mathrm{e}}\left(H_{0}\right)$.

Lemmas 2.3 and 2.4 and the discussion made in the preceding paragraphs enable one to prove the

Theorem 2.6. i) $\rho(H)$ and $\rho\left(H^{*}\right)$ are non-empty and contained in $\rho\left(H_{0}\right) . \quad z \in \rho\left(H_{0}\right)$ belongs to $\rho(H)\left[\rho\left(H^{*}\right)\right]$ if and only if $\sqrt{z}$ is not $Q$ $\left[Q^{*}\right]$ exceptional.

ii) If $z \in \rho(H)\left[\rho\left(H^{*}\right)\right]$, then $R(z)\left[R^{*}(z)\right]$ is given by $(2.6)\left[(2.6)^{*}\right]$.

iii) $\rho\left(H^{*}\right)=\overline{\rho(H)} \equiv\{\bar{z} \in \mathbb{C} \mid z \in \rho(H)\}$.

iv) $R(z)^{*}=R^{*}(\bar{z})$ for $z \in \rho(H)$.

v) $\sigma_{\mathrm{r}}(H)=\sigma_{\mathrm{r}}\left(H^{*}\right)=\phi$.

vi) $\sigma_{\mathrm{e}}(H)=\sigma_{\mathrm{e}}\left(H^{*}\right)=\sigma_{\mathrm{e}}\left(H_{0}\right)$.

vii) $z$ is an eigenvalue of $H$ if and only if $\bar{z}$ is an eigenvalue of $H^{*}$. Neither $H$ nor $H^{*}$ has no non-negative eigenvalues. (Hence, every eigenvalue of $H\left[H^{*}\right]$ has its square root $Q-\left[Q^{*}\right]$ exceptional.) Every 
eigenvalue of $H\left[H^{*}\right]$ has finite multiplicity.

Concerning vii) of the above theorem it should be remarked that 0 is non-exceptional, hence 0 cannot be an eigenvalue of $H$ or $H^{*}$, and that the non-existence of positive eigenvalues follows from a result of Kato [8], for instance. But the latter does not exclude possible (non-zero) real exceptional points.

\section{§ 3. Generalized Eigenfunctions}

It is known that $H_{0}=-\Delta$ has generalized eigenfunctions $\varphi_{0}(x, \xi)=$ $\mathrm{e}^{i\langle\xi, x\rangle}$, where $\langle\xi, x\rangle$ denotes the usual scalar product of 3 -vectors $\xi$ and $x$, coresponding to generalized eigenvalues $|\xi|^{2}$, in terms of which the ordinary Fourier expansion is possible. Here, particular types of generalized eigenfunctions will be constructed for $H$ and $H^{*}$ which are asymptotically equal to $\varphi_{0}(x, \xi)$.

Let $\xi \in \mathbf{R}^{3}$. Consider the functions $\varphi(x, \xi, \kappa)\left[\varphi^{*}(x, \xi, \kappa)\right]$ satisfying the equation

$$
\begin{aligned}
\left(N H_{0}-\kappa^{2}\right) \varphi(x, \xi, \kappa) & =\left(|\xi|^{2}-\kappa^{2}\right) \varphi_{0}(x, \xi) \\
{\left[\left(H_{0} N^{*}-\kappa^{2}\right) \varphi^{*}(x, \xi, \kappa)\right.} & \left.=\left(|\xi|^{2}-\kappa^{2}\right) \varphi_{0}(x, \xi)\right],
\end{aligned}
$$

so that $\varphi(x, \xi, \kappa)\left[\varphi^{*}(x, \xi, \kappa)\right]$ is expected to turn into an eigenfunction of $H\left[H^{*}\right]$ with eigenvalue $|\xi|^{2}$ when $\kappa=|\xi|^{2}$. If $\varphi(x, \xi, \kappa)\left[\varphi^{*}(x, \xi\right.$, $\kappa)]$ has the form

$$
\begin{gathered}
\varphi(x, \xi, \kappa)=\varphi_{0}(x, \xi)+x(x, \xi, \kappa) \\
{\left[\varphi^{*}(x, \xi, \kappa)=N^{*-1} \varphi_{0}(x, \xi)+\chi^{*}(x, \xi, \kappa)\right],}
\end{gathered}
$$

then $x(x, \xi, \kappa)\left[\chi^{*}(x, \xi, \kappa)\right]$ must satisfy the equation

$$
\begin{gathered}
\left(N H_{0}-\kappa^{2}\right) x(x, \xi, \kappa)=|\xi|^{2}(1-N) \varphi_{0}(x, \xi) \\
{\left[\left(H_{0} N^{*}-\kappa^{2}\right) \chi^{*}(x, \xi, \kappa)=\kappa^{2}\left(1-N^{*}\right) N^{*-1} \varphi_{0}(x, \xi)\right] .}
\end{gathered}
$$

Operating formally with $R\left(\kappa^{2}\right)\left[R^{*}\left(\kappa^{2}\right)\right]$ from left and utilizing (2.6) 
$\left[(2.6)^{*}\right]$ yield

$$
\begin{aligned}
x(x, \xi, \kappa)= & |\xi|^{2} R_{0}\left(\kappa^{2}\right) B A \varphi_{0}(x, \xi) \\
& +|\xi|^{2} \kappa^{2} R_{0}\left(\kappa^{2}\right) B(1-Q(\kappa))^{-1} A R_{0}\left(\kappa^{2}\right) B A \varphi_{0}(x, \xi) \\
{\left[x^{*}(x, \xi, \kappa)=\right.} & \kappa^{2} N^{*-1} R_{0}\left(\kappa^{2}\right) B^{*} A \varphi_{0}(x, \xi) \\
& \left.+\kappa^{4} N^{*-1} R_{0}\left(\kappa^{2}\right) A\left(1-Q^{*}(\kappa)\right)^{-1} B^{*} R_{0}\left(\kappa^{2}\right) B^{*} A \varphi_{0}(x, \xi)\right] .
\end{aligned}
$$

In view of the above heuristic consideration a series of functions are defined as follows.

Definition 3.1. Let $x \in \mathbb{R}^{3}, \xi \in \mathbf{R}^{3}$ and $\kappa \notin \tilde{\Sigma}\left[\kappa \notin \tilde{\Sigma}^{*}\right]$.

$$
\begin{aligned}
& \chi_{1}(x, \xi, \kappa)=\frac{|\xi|^{2}}{4 \pi} \int \frac{\mathrm{e}^{\mathrm{i} \kappa|x-y|}}{|x-y|} B(y) A(y) \varphi_{0}(y, \xi) d y_{9}{ }^{3)} \\
& \chi_{1}^{*}(x, \xi, \kappa)=\frac{\kappa^{2}}{4 \pi} \int \frac{\mathrm{e}^{\mathrm{i} \kappa|x-y|}}{|x-y|} B^{*}(y) A(y) \varphi_{0}(y, \xi) d y, \\
& \chi_{2}(x, \xi, \kappa)=\frac{\kappa^{2}}{4 \pi} \int \frac{\mathrm{e}^{\mathrm{i} \kappa|x-y|}}{|x-y|} B(y)\left((1-Q(\kappa))^{-1} A x_{1}\right)(y, \xi, \kappa) d y, \\
& \chi_{2}^{*}(x, \xi, \kappa)=\frac{\kappa^{2}}{4 \pi} \int \frac{\mathrm{e}^{\mathrm{i} \kappa|x-y|}}{|x-y|} A(y)\left(\left(1-Q^{*}(\kappa)\right)^{-1} B^{*} \chi_{1}^{*}\right)(y, \xi, \kappa) d y, \\
& x(x, \xi, \kappa)=x_{1}(x, \xi, \kappa)+x_{2}(x, \xi, \kappa), \\
& \chi^{*}(x, \xi, \kappa)=\overline{n(x)}^{2}\left\{\chi_{1}^{*}(x, \xi, \kappa)+\chi_{2}^{*}(x, \xi, \kappa)\right\}, \\
& \varphi(x, \xi, \kappa)=\varphi_{0}(x, \xi)+x(x, \xi, \kappa), \\
& \varphi^{*}(x, \xi, \kappa)=\overline{n(x)}^{2} \varphi_{0}(x, \xi)+\chi^{*}(x, \xi, \kappa) .
\end{aligned}
$$
cause $\varphi_{0}(x, \xi)$ is bounded and $A(x)$ and $B(x)$ are bounded and of compact support. $\quad \chi_{2}(x, \xi, \kappa)\left[\chi_{2}^{*}(x, \xi, \kappa)\right]$ is well-defined for $(x, \xi, \kappa) \in \mathbf{R}^{3} \times \mathbb{R}^{3}$ $\times(\mathbb{C}-\tilde{\Sigma})\left[\in \mathbb{R}^{3} \times \mathbb{R}^{3} \times\left(\mathbb{C}-\tilde{\Sigma}^{*}\right)\right]$, because, as can be seen easily, $\left(A x_{1}\right)$

3) Here and in the sequel integrals with integration domain unspecified are extended over $\mathbb{R}^{3}$. 
$(\cdot, \xi, \kappa)\left[\left(B^{*} \chi_{1}^{*}\right)(\cdot, \xi, \kappa)\right]$ is in $\mathbf{H}$ and $(1-Q(\kappa))^{-1}\left[\left(1-Q^{*}(\kappa)\right)^{-1}\right]$ is a bounded operator for $\kappa \in \mathbb{C}-\tilde{\Sigma}\left[\kappa \in \mathbb{C}-\tilde{\Sigma}^{*}\right]$ (see lemmas 2.3 and 2.4). It may be checked by straightforward calculation that $x_{1}(x, \xi, \kappa)\left[x_{1}^{*}(x, \xi, \kappa)\right]$ and its first-order $x$-derivatives are continuous in $(x, \xi, \kappa)$. Similarly for $\chi_{2}(x, \xi, \kappa)\left[\chi_{2}^{*}(x, \xi, \kappa)\right]$. Moreover, $\chi_{1}(x, \xi, \kappa)\left[x_{1}^{*}(x, \xi, \kappa)\right]$ has locally $L_{2}$ (distribution) derivatives in $x$ of second order, which follows from the fact that $\chi_{1}(x, \xi, \kappa)\left[x_{1}^{*}(x, \xi, \kappa)\right]$ is essentially a Newtonian potential due to an $L_{2}$ density distribution. Similarly for $\chi_{2}(x, \xi, \kappa)\left[x_{2}^{*}(x, \xi, \kappa)\right]$.

From the above observation a more precise statement will follow in the form of the following

Theorem 3.2. In this theorem $K_{x}$ and $K_{\xi}$ will denote arbitrary compact domains of $\mathbf{R}^{3}, D$ an arbitrary compact domain of $\mathbf{C}-\tilde{\Sigma}\left[\mathbf{C}-\tilde{\Sigma}^{*}\right]$, and $j$ will stand for 1 and 2 .

$\chi_{j}(x, \xi, \kappa)\left[x_{j}^{*}(x, \xi, \kappa)\right]$ is (uniformly) continuous for $(x, \xi, \kappa) \in K_{x} \times$ $K_{\xi} \times D . \quad \chi_{1}(x, \xi, \kappa)\left[x_{1}^{*}(x, \xi, \kappa)\right]$ is smooth in $\xi$ and $\kappa . \chi_{2}(x, \xi, \kappa)\left[x_{2}^{*}(x\right.$, $\xi, \kappa)]$ is smooth in $\xi$ and $\kappa$ for $(\xi, \kappa) \in \mathbf{R}^{3} \times(\mathbf{C}-\tilde{\Sigma})\left[\mathbf{R}^{3} \times\left(\mathbf{C}-\tilde{\Sigma}^{*}\right)\right]$. The first-order $x$-derivatives of $\chi_{j}(x, \xi, \kappa)\left[x_{j}^{*}(x, \xi, \kappa)\right]$ are (uniformly) continuous for $(x, \xi, \kappa) \in K_{x} \times K_{\xi} \times D . \quad \chi_{j}(x, \xi, \kappa)\left[x_{j}^{*}(x, \xi, \kappa)\right]$ has locally $L_{2} x$-derivatives in the distribution sense which are continuous in the local $L_{2}$ topology for $(\xi, \kappa) \in K_{\xi} \times D . \varphi(x, \xi, \kappa)\left[\varphi^{*}(x, \xi, \kappa)\right]$ and $x(x, \xi, \kappa)$ $\left[x^{*}(x, \xi, \kappa)\right]$ satisfy equations (3.1) and (3.2) $\left[(3.1)^{*}\right.$ and $\left.(3.2)^{*}\right]$, respectively, in the distribution sense.

The eigenfunction $\varphi_{0}(x, \xi)$ is a plane wave with $\xi$ the wave vector. The functions $\varphi(x, \xi, \kappa)$ and $\varphi^{*}(x, \xi, \kappa)$ for non-real $\kappa$ represent what has been referred to in the introduction as the off-real distorted plane waves, as is clearly suggested in definition 3.1. The distorted plane waves $\varphi(x, \xi, \kappa)$ and $\varphi^{*}(x, \xi, \kappa)$ with $\kappa= \pm|\xi|$ may deserve a special notation in the light of the rôle played later by them in spectral representation.

\section{Definition 3.3.}

$$
\begin{array}{ll}
\varphi_{ \pm}(x, \xi)=\varphi(x, \xi, \pm|\xi|) & \text { for } \pm|\xi| \notin \Sigma \subset \tilde{\Sigma}, \\
\varphi_{ \pm}^{*}(x, \xi)=\varphi^{*}(x, \xi, \pm|\xi|) & \text { for } \pm|\xi| \notin \Sigma^{*} \subset \tilde{\Sigma}^{*} .
\end{array}
$$


As is seen from equation (3.1) $\left[(3.1)^{*}\right]$ with $\kappa= \pm|\xi|, \varphi_{ \pm}(x, \xi)$ $\left[\varphi_{ \pm}^{*}(x, \xi)\right]$ is a generalized eigenfunction with eigenvalue $|\xi|^{2}$ of $H=$ $N H_{0}=-n^{-2} \Delta\left[H^{*}=H_{0} N^{*}=-\Delta n^{-2}\right]$, and may well be called a distorted plane , wave.

\section{§4. Representation of the Resolvents}

This section derives a representation for $R(z)$ and $R^{*}(z)$ in terms of Fourier transforms in line with Shenk [5].

Let $\kappa$ be such that $\operatorname{Im} \kappa>0$ and $\kappa^{2} \in \rho(H)\left[\rho\left(H^{*}\right)\right]$. Let $f \in C_{0}^{\infty}\left(\mathbb{R}^{3}\right)$, i.e., let $f$ be of class $C^{\infty}$ with compact support, and let $\hat{f}_{0}$ denote the Fourier transform of $f$ :

$$
\hat{f}_{0}(\xi)=(2 \pi)^{-3 / 2} \sqrt{\varphi_{0}(x, \xi)} f(x) d x
$$

Define the functions

$$
\begin{aligned}
& \Phi(x, \kappa, f)=(2 \pi)^{-3 / 2} \int\left(|\xi|^{2}-\kappa^{2}\right)^{-1} \varphi(x, \xi, \kappa) \hat{f}_{0}(\xi) d \xi \\
& \Phi^{*}(x, \kappa, f)=(2 \pi)^{-3 / 2} \int\left(|\xi|^{2}-\kappa^{2}\right)^{-1} \varphi^{*}(x, \xi, \kappa) \hat{f}_{0}(\xi) d \xi
\end{aligned}
$$

Since $\hat{f}_{0}(\xi)$, as the Fourier transform of a $C_{0}^{\infty}$ function, is smooth and rapidly decreasing, and since, as is seen from definition $3.1, \varphi(x, \xi, \kappa)$ and $\varphi^{*}(x, \xi, \kappa)$ are at most of polynomial growth in $|\xi|$, the above functions are well-defined. Moreover, $\operatorname{Im} \kappa>0$ implies $\Phi(\bullet, \kappa, f) \in D(H)$ and $\Phi^{*}(\cdot$, $\kappa, f) \in D\left(H^{*}\right)$ on account of theorem 3.2 and of the fact that $x_{j}$ and $x_{j}^{*}$, as set forth in definition 3.1, have $R_{0}\left(\kappa^{2}\right)$ as the leftmost factor. Equations (3.1) and $\left(3.1^{*}\right)$ fulfilled by $\varphi(x, \xi, \kappa)$ and $\varphi^{*}(x, \xi, \kappa)$ (theorem 3.2) and the inversion formula for Fourier transforms give rise to the equations

$$
\left(H-\kappa^{2}\right) \Phi(x, \kappa, f)=f(x) \text { and }\left(H^{*}-\kappa^{2}\right) \Phi^{*}(x, \kappa, f)=f(x) .
$$

The preceding argument yields the following 
Theorem 4.1. For $f \in C_{0}^{\infty}\left(\mathbf{R}^{3}\right)$ and $\kappa$ such that $\operatorname{Im} \kappa>0$ and $\kappa^{2} \in$ $\rho(H)\left[\kappa^{2} \in \rho\left(H^{*}\right)\right]$

$$
\begin{aligned}
& \left(R\left(\kappa^{2}\right) f\right)(x)=(2 \pi)^{-3 / 2} \int\left(|\xi|^{2}-\kappa^{2}\right)^{-1} \varphi(x, \xi, \kappa) \hat{f}_{0}(\xi) d \xi \\
& {\left[\left(R^{*}\left(\kappa^{2}\right) f\right)(x)=(2 \pi)^{-3 / 2} \int\left(|\xi|^{2}-\kappa^{2}\right)^{-1} \varphi^{*}(x, \xi, \kappa) \hat{f}_{0}(\xi) d \xi\right] .}
\end{aligned}
$$

\section{§. Spectral Measures}

Let $E_{0}$ be the spectral measure (resolution of the identity) associated with the unperturbed self-adjoint operator $H_{0}$ so that $\left(E_{0}(\Delta) f, g\right)$ with $\Delta$ a bounded Borel set of $\mathbb{R}^{1}$ and $f, g \in \mathbf{H}$ may be given the form

$$
\begin{aligned}
\left(E_{0}(\Delta) f, g\right) & =\frac{1}{2 \pi \mathrm{i}} \lim _{\varepsilon \downarrow 0} \int_{\Delta}\left(R_{0}(\lambda+\mathrm{i} \varepsilon) f-R_{0}(\lambda-\mathrm{i} \varepsilon) f, g\right) d \lambda \\
& =\lim _{\varepsilon \downarrow 0} \frac{\varepsilon}{\pi} \int_{\Delta}\left(R_{0}(\lambda \pm \mathrm{i} \varepsilon) f, R_{0}(\lambda \pm \mathrm{i} \varepsilon) g\right) d \lambda,
\end{aligned}
$$

where and in the sequel the (first) resolvent equation $R\left(z_{1}\right)-R\left(z_{2}\right)=$ $\left(z_{1}-z_{2}\right) R\left(z_{1}\right) R\left(z_{2}\right)$ is used without explicit mention. The purpose of the present section is to define a (not necessarily orthogonal projection valued) spectral measure $E\left[E^{*}\right]$ for $H\left[H^{*}\right]$ in analogy with formula (5.1) by the following relation:

$$
\begin{aligned}
(E .2) \quad(\Delta) f, g) & =\frac{1}{2 \pi \mathrm{i}} \lim _{\varepsilon \downarrow 0} \int_{\Delta}(R(\lambda+\mathrm{i} \varepsilon) f-R(\lambda-\mathrm{i} \varepsilon) f, g) d \lambda \\
& =\lim _{\varepsilon \downarrow 0} \frac{\varepsilon}{\pi} \int_{\Delta}\left(R(\lambda \pm \mathrm{i} \varepsilon) f, R^{*}(\lambda \pm \mathrm{i} \varepsilon) g\right) d \lambda \\
(5.2)^{*} \quad\left[\left(E^{*}(\Delta) f, g\right)\right. & =\frac{1}{2 \pi \mathrm{i}} \lim _{\varepsilon \downarrow 0} \int_{\Delta}\left(R^{*}(\lambda+\mathrm{i} \varepsilon) f-R^{*}(\lambda-\mathrm{i} \varepsilon) f, g\right) d \lambda \\
& \left.=\lim _{\varepsilon \downarrow 0} \frac{\varepsilon}{\pi} \int_{\Delta}\left(R^{*}(\lambda \pm \mathrm{i} \varepsilon) f, R(\lambda \pm \mathrm{i} \varepsilon) g\right) d \lambda\right],
\end{aligned}
$$

where theorem 2.6, iv) has been used. If these definitions make sense, 
then clearly

$$
E(\Delta)^{*}=E^{*}(\Delta)
$$

For a moment (5.2) will be considered exclusively, for (5.2)* may be obtained by the complex conjugation of (5.2) with $f$ and $g$ exchanged. By use of (5.1) and the resolvent equation (2.1) the right-hand side of (5.2) can be rewritten as

$$
\begin{aligned}
& \left(E_{0}(\Delta) N^{-1} f, g\right)+\frac{1}{2 \pi \mathrm{i}} \lim _{\varepsilon \downarrow 0} \int_{\Delta} \lambda\left(A R(\lambda+\mathrm{i} \varepsilon) f, B^{*} R_{0}(\lambda-\mathrm{i} \varepsilon) g\right) d \lambda \\
& -\frac{1}{2 \pi \mathrm{i}} \lim _{\varepsilon \downarrow 0} \int_{\Delta} \lambda\left(A R(\lambda-\mathrm{i} \varepsilon) f, B^{*} R_{0}(\lambda+\mathrm{i} \varepsilon) g\right) d \lambda \\
& +\lim _{\varepsilon \downarrow 0} \frac{\varepsilon}{2 \pi} \int_{\Delta}\left[\left(A R(\lambda+\mathrm{i} \varepsilon) f, B^{*} R_{0}(\lambda-\mathrm{i} \varepsilon) g\right)\right. \\
& \left.+\left(A R(\lambda-\mathrm{i} \varepsilon) f, B^{*} R_{0}(\lambda+\mathrm{i} \varepsilon) g\right)\right] d \lambda .
\end{aligned}
$$

According to Kato [9] and Mochizuki [1] the following lemma holds.

Lemma 5.1. Operators $A$ and $B$ are $H_{0}$-smooth, i.e., if $T$ stands for $A$ or $B$, then for any $f \in \mathbb{H}$

$$
\int_{0}^{\infty}\left\|T R_{0}(\lambda \pm \mathrm{i} \varepsilon) f\right\|^{2} d \lambda \leqq C\|f\|^{2}
$$

where $C$ is a constant independent of $\varepsilon$ and $f$. If $\Delta$ is a bounded Borel set of $[0, \infty)$ such that $\pm \sqrt{\Delta} \equiv\{ \pm \sqrt{\lambda} \mid \lambda \in \Delta\}$ is at a positive distance from $\Sigma$, then $T=A, B$ is $H$-smooth:

$$
\int_{\Delta}\|T R(\lambda \pm \mathrm{i} \varepsilon) f\|^{2} d \lambda \leqq C\|f\|^{2}
$$

where $C$ is a constant independent of $\varepsilon$ and $f$.

By the above lemma it follows that if the Borel set $\Delta$ is chosen as indicated in the lemma, then each term of (5.4) is meaningful, and, in particular, the last term vanishes. Thus expression (5.4) or the righthand side of (5.2) defines a bounded bilinear form on $\mathbb{H}$, which in turn 
implies by virtue of F. Riesz' theorem the existence of a unique bounded linear operator $E(\Delta)$ such that equation (5.2) is valid. $E(\Delta)$ is called the spectral measure associated with $H$. As remarked before, the spectral measure $E^{*}$ associated with $H^{*}$ also turns out well-defined so that $(5.2)^{*}$ holds.

In quite a similar way one can define bounded linear operators $U_{ \pm}(\Delta)$ and $V_{ \pm}(\Delta)$ by

$$
\begin{aligned}
& \left(U_{ \pm}(\Delta) f, g\right)=\lim _{\varepsilon \downarrow 0} \frac{\varepsilon}{\pi} \int_{\Delta}\left(R_{0}(\lambda \mp \mathrm{i} \varepsilon) f, R^{*}(\lambda \mp \mathrm{i} \varepsilon) g\right) d \lambda, \\
& \left(V_{ \pm}(\Delta) f, g\right)=\lim _{\varepsilon \downarrow 0} \frac{\varepsilon}{\pi} \int_{\Delta}\left(R(\lambda \mp \mathrm{i} \varepsilon) f, R_{0}(\lambda \mp \mathrm{i} \varepsilon) g\right) d \lambda .
\end{aligned}
$$

The reason why $E(\Delta)$ and $E^{*}(\Delta)$ bear the name of spectral measure will be clarified more in the following section.

Summing up, one arrives at the

Theorem 5.2. Let $\Delta$ be a bounded Borel set of $[0, \infty)$ such that both $\sqrt{\Delta}$ and $-\sqrt{\Delta}$ are at a positive distance from $\Sigma$ (or, what comes to the same thing, $\left.\Sigma^{*}\right) .{ }^{4}$ Then the right-hand sides of (5.2), (5.2)*, (5.5) and (5.6) make sense for any $f, g \in \mathbf{H}$, and hence define unique bounded operators $E(\Delta), E^{*}(\Delta), U_{ \pm}(\Delta)$ and $V_{ \pm}(\Delta)$, and relation (5.3) holds.

\section{§6. Spectral Representation}

It is well known that a spectral representation for $H_{0}$ is furnished in terms of Fourier transforms by the formula

$$
\left(E_{0}(\Delta) f, g\right)=\int_{A_{\sqrt{\Delta}}} \hat{f}_{0}(\xi) \overline{\hat{g}_{0}(\xi)} d \xi \equiv\left(\hat{f}_{0}, \hat{g}_{0}\right)_{A_{\sqrt{\Delta}}}
$$

where $A_{\sqrt{\Delta}}=\left\{\xi \in \mathbf{R}^{3}|| \xi \mid \in \sqrt{\Delta}\right\}$. In order to establish a similar formula for $E(\Delta)\left[E^{*}(\Delta)\right]$ a few function(al)s are to be defined first.

4) In fact $\tilde{\Sigma}$ and $\tilde{\Sigma}^{*}$, a fortiori $\Sigma$ and $\Sigma^{*}$, are symmetric in the origin 0 , which is a consequence of the relation $Q^{*}(\kappa)=Q(-\bar{\kappa})^{*}$ (see below (2.5)*). 
Definition 6.1. Let $f \in C_{0}^{\infty}\left(\mathbb{R}^{3}\right)$. Let $\varphi(x, \xi, \kappa), \varphi^{*}(x, \xi, \kappa), \varphi_{ \pm}(x$, $\xi)$ and $\varphi_{ \pm}^{*}(x, \xi)$ be as in definitions 3.1 and 3.3. Define

$$
\begin{aligned}
& \hat{f}(\xi, \kappa)=(2 \pi)^{-3 / 2} \int \overline{\varphi(x, \xi, \kappa)} f(x) d x \\
& \hat{f}^{*}(\xi, \kappa)=(2 \pi)^{-3 / 2} \int \overline{\varphi^{*}(x, \xi, \kappa)} f(x) d x \\
& \hat{f}_{ \pm}(\xi)=\hat{f}(\xi, \pm|\xi|)=(2 \pi)^{-3 / 2} \sqrt{\varphi_{ \pm}(x, \xi)} f(x) d x \\
& \hat{f}_{ \pm}^{*}(\xi)=\hat{f}^{*}(\xi, \pm|\xi|)=(2 \pi)^{-3 / 2} \int \overline{\varphi_{ \pm}^{*}(x, \xi)} f(x) d x
\end{aligned}
$$

Lemma 6.2. Let $f \in C_{0}^{\infty}\left(\mathbf{R}^{3}\right)$, and let $\kappa$ be such that $\operatorname{Im} \kappa>0$ and $\kappa^{2} \in \rho(H)\left[\kappa^{2} \in \rho\left(H^{*}\right)\right]$. Then

$$
\begin{gathered}
\left(R\left(\kappa^{2}\right) f\right)_{0}(\xi)=\left(|\xi|^{2}-\kappa^{2}\right)^{-1} \hat{f}^{*}(\xi,-\bar{\kappa}) \\
{\left[\left(R^{*}\left(\kappa^{2}\right) f\right)_{0}(\xi)=\left(|\xi|^{2}-\kappa^{2}\right)^{-1} \hat{f}(\xi,-\bar{\kappa})\right] .}
\end{gathered}
$$

Proof. Theorem 4.1, definition 6.1 and the Parseval formula for ordinary Fourier transforms enable one to write

$$
\begin{gathered}
\left(\left(R\left(\kappa^{2}\right) \hat{f}\right)_{0}, g_{0}\right)=\left(R\left(\kappa^{2}\right) f, g\right)=\left(f, R^{*}\left((-\bar{\kappa})^{2}\right) g\right)=\left(f, \Phi^{*}(\cdot,-\bar{\kappa}, g)\right) \\
=(2 \pi)^{-3 / 2} \iint f(x)\left(|\xi|^{2}-\kappa^{2}\right)^{-1} \overline{\varphi^{*}(x, \xi,-\bar{\kappa}) \hat{g}_{0}(\xi)} d x d \xi \\
=\left(\left(|\cdot|^{2}-\kappa^{2}\right)^{-1} \hat{f}^{*}(\cdot,-\bar{\kappa}), g_{0}\right) \\
{\left[\left(\left(R^{*}\left(\kappa^{2}\right) f\right)_{0}, g_{0}\right)=\left(\left(|\cdot|^{2}-\kappa^{2}\right)^{-1} \hat{f}(\cdot,-\bar{\kappa}), g_{0}\right)\right] \cdot}
\end{gathered}
$$

Taking into account the arbitrariness of $g \in C_{0}^{\infty}\left(\mathbf{R}^{3}\right)$ yields the lemma.

Q.E.D.

Let $\Delta$ be as in theorem 5.2. It is then possible by virtue of theorem 5.2 and the above lemma 6.2 to calculate $(E(\Delta) f, g), f, g \in C_{0}^{\infty}\left(\mathbb{R}^{3}\right)$, as follows: 


$$
\begin{aligned}
(E(\Delta) f, g) & =\lim _{\varepsilon \downarrow 0} \frac{\varepsilon}{\pi} \int_{\Delta}\left(R(\lambda \mp \mathrm{i} \varepsilon) f_{9} R^{*}(\lambda \mp \mathrm{i} \varepsilon) g\right) d \lambda \\
& =\lim _{\varepsilon \downarrow 0} \frac{\varepsilon}{\pi} \int_{\Delta}\left(\int(R(\lambda \mp \mathrm{i} \varepsilon) f)_{0}^{\wedge}(\xi) \overline{\left(R^{*}(\lambda \mp \mathrm{i} \varepsilon) g\right)_{0}^{\wedge}(\xi)} d \xi\right) d \lambda \\
& =\lim _{\varepsilon \downarrow 0} \frac{\varepsilon}{\pi} \int_{\Delta}\left(\int \frac{1}{\left(|\xi|^{2}-\lambda\right)^{2}+\varepsilon^{2}} \hat{f}^{*}(\xi,-\sqrt{\lambda \mp \mathrm{i} \varepsilon} \overline{\hat{g}(\xi,-\sqrt{\lambda \mp i \varepsilon})} d \xi) d \lambda .\right.
\end{aligned}
$$

In view of theorem 3.2 and the fact that $f, g$ are smooth with compact support one can see that $\hat{f}^{*}(\xi,-\sqrt{\lambda \mp \mathrm{i} \varepsilon})$ and $\left(1+|\xi|^{2}\right)^{-1} \hat{g}(\xi,-\sqrt{\lambda \mp \mathrm{i} \varepsilon})$ are bounded continuous functions of $\xi, \lambda$ and $\varepsilon$ for $(\xi, \lambda, \varepsilon) \in \mathbb{R}^{3} \times \Delta \times[0$, $\left.\varepsilon_{0}\right)\left(\varepsilon_{0}>0\right)$. It can also be checked that these functions are square integrable for large $|\xi|$ uniformly in $(\lambda, \varepsilon) \in \Delta \times\left[0, \varepsilon_{0}\right)$. Therefore, one can freely interchange the order of limit and integration in the above expression, and then make use of the symbolic relation

$$
\lim _{\varepsilon \downarrow 0} \frac{\varepsilon}{\pi} \frac{1}{(\mu-\lambda)^{2}+\varepsilon^{2}}=\delta(\mu-\lambda)
$$

$\delta$ denoting the Dirac delta function, to obtain by definition 6.1

$$
(E(\Delta) f, g)=\int_{|\xi| \in \sqrt{\Delta}} \hat{f}_{ \pm}^{*}(\xi) \overline{\hat{g}_{ \pm}(\xi)} d \xi
$$

(For a more detailed argument see $[1]$ or $[4]$.) One can also obtain a similar formula for $\left(E^{*}(\Delta) f, g\right)$ in view of theorem 5.2. Thus the following lemma results.

Lemma 6.3. Let $f, g \in C_{0}^{\infty}\left(\mathbb{R}^{3}\right)$ and let $\Delta$ be as in theorem 5.2. Then

$$
(E(\Delta) f, g)=\left(\hat{f}_{ \pm}^{*}, \hat{g}_{ \pm}\right)_{A_{\sqrt{\Delta}}} \text { and }\left(E^{*}(\Delta) f, g\right)=\left(\hat{f}_{ \pm}, \hat{g}_{ \pm}^{*}\right)_{A_{\sqrt{\Delta}}} .
$$

If in $\hat{f}_{ \pm}(\xi)$ or $\hat{f}_{ \pm}^{*}(\xi)$ the argument $\xi$ is restricted to $A_{\sqrt{\Delta}}$, then $\hat{~}_{ \pm}$or ^ \pm may be regarded as a linear operator from $C_{0}^{\infty}\left(\mathbb{R}^{3}\right) \subset \mathbb{H}$ to $L_{2}\left(A_{\sqrt{\Delta}}\right)$. Its boundedness is not yet known, however.

Lemma 6.4. Let $\Delta$ be as in theorem 5.2. Then the operators $\hat{A}_{ \pm}$ 
and $\wedge^{\wedge}$ 类 are (able to be extended to) bounded linear operators on $\mathbb{H}^{\mathrm{H}}$ to $L_{2}\left(A_{\sqrt{4}}\right)$, and

$$
\begin{aligned}
& \hat{f}_{ \pm}(\xi)=\left(U_{ \pm}(\Delta)^{*} f\right)^{\wedge}{ }_{0}(\xi) \quad \text { for a.e. } \xi \in A_{\sqrt{\Delta}} \text {, } \\
& \hat{f}_{ \pm}^{*}(\xi)=\left(V_{ \pm}(\Delta) f\right)^{\wedge}{ }_{0}(\xi) \quad \text { for a.e. } \xi \in A_{\sqrt{\Delta}},
\end{aligned}
$$

where the $U_{ \pm}(\Delta)$ and $V_{ \pm}(\Delta)$ are bounded linear operators on $\mathbb{H}$ introduced in the preceding section.

Proof. First let $f, g \in C_{0}^{\infty}\left(\mathbb{R}^{3}\right)$. Arguing as in the proof of the preceding lemma brings forth

$$
\left(U_{ \pm}(\Delta) * f, g\right)=\left(f, U_{ \pm}(\Delta) g\right)=\left(\hat{f}_{ \pm}, \hat{g}_{0}\right)_{A_{\sqrt{4}}} .
$$

Since the Fourier image of $C_{0}^{\infty}\left(\mathbb{R}^{3}\right)$ is dense in $L_{2}\left(\mathbb{R}^{3}\right)$,

$$
\left(U_{ \pm}(\Delta) * f\right)^{\wedge}{ }_{0}(\xi)=\hat{f}_{ \pm}(\xi) \text { for } \xi \in A_{\sqrt{\Delta}} ;=0 \text { for } \xi \notin A_{\sqrt{\Delta}} .
$$

From this follow the assertions concerning ${ } \pm$ owing to the boundedness of $U_{ \pm}(\Delta)$ and the Fourier transformation and to the density of $C_{0}^{\infty}\left(\mathbb{R}^{3}\right)$ in H. The assertions concerning ${ }^{\wedge} *$ can be proved similarly. $\quad$ Q.E.D.

Incidentally in the above proof has been shown the next

Lemma 6.5. Let $\Delta$ be as in theorem 5.2. Then for $f \in \mathbb{H}$

$$
\begin{aligned}
& \left(U_{ \pm}(\Delta)^{*} f\right)^{\wedge}{ }_{0}(\xi)=\left\{\begin{array}{cl}
\hat{f}_{ \pm}(\xi) & \text { for a.e. } \xi \in A_{\sqrt{\bar{A}}} \\
0 & \text { for a.e. } \xi \notin A_{\sqrt{\bar{A}}},
\end{array}\right. \\
& \left(V_{ \pm}(\Delta) f\right)^{\wedge}{ }_{0}(\xi)=\left\{\begin{array}{cl}
\hat{f}_{ \pm}^{*}(\xi) & \text { for a.e. } \xi \in A_{\sqrt{\Delta}} \\
0 & \text { for a.e. } \xi \notin A_{\sqrt{\Delta}} .
\end{array}\right.
\end{aligned}
$$

From lemmas 6.3 and 6.4 (or 6.5) and the Parseval formula readily follows the expression for $E(\Delta)$ in terms of $U_{ \pm}(\Delta)$ and $V_{ \pm}(\Delta)$.

Lemma 6.6. $E(\Delta)=U_{ \pm}(\Delta) V_{ \pm}(\Delta)$, where $\Delta$ is as in theorem 5.2 . 
The following lemma is not very hard, and can be proved by using lemmas 6.3 and 6.4, the closedness of $H$ and $H^{*}$ and the properties of the generalized eigenfunctions $\varphi_{ \pm}(x, \xi)$ and $\varphi_{ \pm}^{*}(x, \xi)$ stated in theorem 3.2 .

Lemma 6.7. Let $\Delta$ be as in theorem 5.2. If $f \in D(H)\left[D\left(H^{*}\right)\right]$,

$$
(H f)^{\wedge} \stackrel{*}{ \pm}=|\cdot|^{2} \hat{f}_{ \pm}^{*} \quad\left[\left(H^{*} f\right)^{\wedge}{ }_{ \pm}=|\cdot|^{2} \hat{f}_{ \pm}\right] \quad \text { in } L_{2}\left(A_{v^{\prime}}\right) \text {. }
$$

$(E(e) f, g)\left[\left(E^{*}(e) f, g\right)\right]$, where $f, g \in \mathbf{H}$ and $e$ varies over all Borel subsets of $A$, is a bounded signed measure absolutely continuous with respect to the ordinary Lebesgue measure, and if in addition $f \in D(H)\left[D\left(H^{*}\right)\right]$,

$$
\begin{gathered}
(E(e) H f, g)=\int_{e} \lambda d(E(\lambda) f, g)=\left(|\cdot|^{2} \hat{f}_{ \pm}^{*}, \hat{g}_{ \pm}\right)_{A_{\sqrt{ }_{e}}}=(H E(e) f, g) \\
{\left[\left(E^{*}(e) H^{*} f, g\right)=\int_{e} \lambda d\left(E^{*}(\lambda) f, g\right)=\left(|\cdot|^{2} \hat{f}_{ \pm}, \hat{g}_{ \pm}^{*}\right)_{A_{\sqrt{e}}}=\left(H^{*} E^{*}(e) f, g\right)\right],}
\end{gathered}
$$

the last two equalities holding without the assumption $f \in D(H)\left[D\left(H^{*}\right)\right]$, and hence $H\left[H^{*}\right]$ and $E(e)\left[E^{*}(e)\right]$ commute.

Using the above lemma one can define a bounded linear operator $H(\Delta)=H E(\Delta)$ which coincides with $H$ on the range of $E(\Delta)$. Similarly, a bounded linear operator $H^{*}(\Delta)=H^{*} E^{*}(\Delta)$ is defined. One can also consider functions of $H(\Delta)$ and $H^{*}(\Delta)$. Let $\alpha$ be a bounded Borel function on $\Delta$. Define $\alpha(H(\Delta))\left[\alpha\left(H^{*}(\Delta)\right)\right]$ by

$$
\begin{gathered}
(\alpha(H(\Delta)) f, g)=\int_{\Delta} \alpha(\lambda) d(E(\lambda) f, g) \\
{\left[\left(\alpha\left(H^{*}(\Delta)\right) f, g\right)=\int_{\Delta} \alpha(\lambda) d\left(E^{*}(\lambda) f, g\right)\right] .}
\end{gathered}
$$

Then on the basis of lemma 6.7 the following result obtains immediately.

Lemma 6.8. Let $\Delta$ be as in theorem 5.2. Let $\alpha$ and $\beta$ be bounded Borel measurable functions on 4 . Then

$$
\alpha \beta(H(\Delta))=\alpha(H(\Delta)) \beta(H(\Delta))=\beta(H(\Delta)) \alpha(H(\Delta)),
$$




$$
\alpha \beta\left(H^{*}(\Delta)\right)=\alpha\left(H^{*}(\Delta)\right) \beta\left(H^{*}(\Delta)\right)=\beta\left(H^{*}(\Delta)\right) \alpha\left(H^{*}(\Delta)\right) .
$$

For a Borel set $e \subset \Delta x_{e}(H(\Delta))=E(e)$ and $\chi_{e}\left(H^{*}(\Delta)\right)=E^{*}(e)$, where $x_{e}$ denotes the characteristic function of $e$. Hence for any $e_{1}, e_{2} \subset \Delta, E\left(e_{1} \cap e_{2}\right)$ $=E\left(e_{1}\right) E\left(e_{2}\right)=E\left(e_{2}\right) E\left(e_{1}\right)$ and $E^{*}\left(e_{1} \cap e_{2}\right)=E^{*}\left(e_{1}\right) E^{*}\left(e_{2}\right)=E^{*}\left(e_{2}\right) E^{*}\left(e_{1}\right)$, and, in particular, $E(e)^{2}=E(e)$ and $E^{*}(e)^{2}=E^{*}(e)$, i.e., $E(e)$ and $E^{*}(e)$ are (not necessarily orthogonal) projections.

In passing it is remarked that lemmas 6.7 and 6.8 and the discussion in between justify naming $E(\Delta)\left[E^{*}(\Delta)\right]$ the spectral measure for $H\left[H^{*}\right]$.

At present it is not known whether $\hat{}_{ \pm}$and $\stackrel{\wedge}{ \pm}$ map onto $L_{2}\left(A_{\sqrt{4}}\right)$. To settle this problem is important in order to establish a unitary spectral representation map.

Lemma 6.9. ${ }^{5)}$ The operators $\hat{\wedge}_{ \pm}$and ${ }^{\wedge}$ 类 map $\mathbf{H}$ onto $L_{2}\left(A_{\sqrt{\Delta}}\right)$, where $\Delta$ is as in theorem 5.2 .

Proof. Suppose ${ }^{ \pm} \pm$is not onto $L_{2}\left(A_{\sqrt{ }}\right)$. Then there exists a nonzero element $u \in L_{2}\left(A_{\sqrt{\Delta}}\right)$ such that for any $f \in C_{0}^{\infty}\left(\mathbf{R}^{3}\right)\left(\hat{f}_{ \pm}, u\right)_{A_{\sqrt{\Delta}}}=0$. This implies

$$
\int_{A_{\sqrt{\overline{4}}}} \varphi_{ \pm}(x, \xi) u(\xi) d \xi=0
$$

Now apply $N H_{0}=-n^{-2} \Delta$ and use theorem 3.2 to obtain

$$
\int_{A_{\sqrt{\bar{\Delta}}}}|\xi|^{2} \varphi_{ \pm}(x, \xi) u(\xi) d \xi=0
$$

A justification of this may be provided by Fubini's theorem. Repeating this procedure gives

$$
\int_{A_{\sqrt{ }, \bar{\Delta}}}|\xi|^{m} \varphi_{ \pm}(x, \xi) u(\xi) d \xi=0
$$

for all non-negative even integers $m$, which in turn yields

5) The lemma may be proved by the method of Mochizuki [1], which consists in a direct verification of lemma 6.12 , from which it is easy to deduce the lemma. 


$$
\int_{|\xi|^{2}=\lambda} \varphi_{ \pm}(x, \xi) u(\xi) d S_{\xi}=0 \quad \text { for a.e. } \lambda \in \sqrt{\Delta}
$$

or, definitions 3.1 and 3.3 being noted,

$$
v(x, \lambda) \equiv \int_{|\xi|^{2}=\lambda} \varphi_{0}(x, \xi) u(\xi) d S_{\xi}=-\int_{|\xi|^{2}=\lambda} x(x, \xi, \pm|\xi|) u(\xi) d S_{\xi}
$$

for a.e. $\lambda \in \sqrt{\Delta}$. It is easy to see that $x(x, \xi, \pm|\xi|)$ satisfies the radiation condition. Then it is possible to argue as in Ikebe [10] (note that $u(\xi)$ may be assumed to be 0 outside $\left.A_{\sqrt{\Delta}}\right)$ to the effect that $u=0$, which is a contradiction. This proves that $\hat{\AA}$ maps onto $L_{2}\left(A_{\sqrt{4}}\right)$. A similar argument applies to $\stackrel{\wedge}{ \pm}$.

Q.E.D.

It follows from lemmas 6.3 and 6.8 that

$$
\left(\hat{f}_{ \pm}^{*}, \hat{g}_{ \pm}\right)_{A_{\sqrt{\Delta_{1}}}}=\left(E\left(\Delta_{1}\right) f, g\right)=\left(E\left(\Delta_{2}\right) E\left(\Delta_{1}\right) f, g\right)=\left(\left(E\left(\Delta_{1}\right) f\right)^{\wedge} \underset{ \pm}{*}, \hat{g}_{ \pm}\right)_{A_{\sqrt{\Delta_{2}}}}
$$

for $\Delta_{1} C \Delta_{2}$. This equation combined with lemma 6.9 gives the following

Lemma 6.10. Let $\Delta$ be as in theorem 5.2. Then

$$
\begin{gathered}
(E(\Delta) f)^{\wedge} \underset{ \pm}{*}(\xi)=\left\{\begin{array}{cc}
\hat{f}_{ \pm}^{*}(\xi) & \text { for a.e. } \xi \in A_{\sqrt{\Delta}} \\
0 & \text { for a.e. } \xi \notin A_{\sqrt{\Delta}},
\end{array}\right. \\
\left(E^{*}(\Delta) f\right)^{\wedge}(\xi)=\left\{\begin{array}{cc}
\hat{f}_{ \pm}(\xi) & \text { for a.e. } \xi \in A_{\sqrt{\Delta}} \\
0 & \text { for a.e. } \xi \notin A_{\sqrt{\Delta}}
\end{array}\right.
\end{gathered}
$$

From lemmas $6.3,6.4,6.9$ and 6.10

$$
\begin{aligned}
\left(U_{ \pm}(\Delta) f, g\right) & =\left(\hat{f}_{0}, \hat{g}_{ \pm}\right)_{A_{\sqrt{\Delta}}}=\left(\hat{f}_{0},\left(E^{*}(\Delta) g\right)^{\wedge}\right)_{A_{\sqrt{\Delta}}}=\left(U_{ \pm}(\Delta) f, E^{*}(\Delta) g\right) \\
& =\left(E(\Delta) U_{ \pm}(\Delta) f, g\right)=\left(\left(U_{ \pm}(\Delta) f\right)^{\wedge} \stackrel{*}{ \pm}, \hat{g}_{ \pm}\right)_{A_{\sqrt{\Delta}}} .
\end{aligned}
$$

The following assertion is entailed by the above equation and the corresponding one involving $V_{ \pm}(\Delta)$.

Lemma 6.11. Let $\Delta$ be as in theorem 5.2. Then 


$$
\begin{array}{ll}
\left(U_{ \pm}(\Delta) f\right)^{\wedge}{ }_{ \pm}^{*}=\left(E_{0}(\Delta) f\right)^{\wedge}, & \left(V_{ \pm}(\Delta)^{*} f\right)^{\wedge}=\left(E_{0}(\Delta) f\right)^{\wedge}, \\
E(\Delta) U_{ \pm}(\Delta)=U_{ \pm}(\Delta), & V_{ \pm}(\Delta) E(\Delta)=V_{ \pm}(\Delta) .
\end{array}
$$

As a direct consequence of the above lemma one obtains the

Lemma 6.12. Let $\Delta$ be as in theorem 5.2. Then $E_{0}(\Delta)=$ $V_{ \pm}(\Delta) U_{ \pm}(\Delta), U_{ \pm}(\Delta) E_{0}(\Delta)=U_{ \pm}(\Delta)$ and $E_{0}(\Delta) V_{ \pm}(\Delta)=V_{ \pm}(\Delta)$.

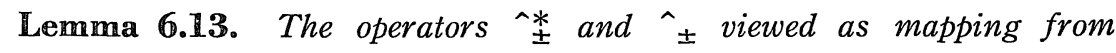
$E(\Delta) \mathbb{H}$ and $E^{*}(\Delta) \mathrm{H}$, respectively, to $L_{2}\left(A_{\sqrt{\Delta}}\right)$ are one-to-one and onto, where $\Delta$ is as in theorem 5.2. (Note that $E(\Delta) \mathbb{H}$ and $E^{*}(\Delta) \mathbf{H}$ are closed linear subspaces of $\mathbb{H}$ because of $E(\Delta)$ and $E^{*}(\Delta)$ being projections (lemma 6.8).)

Proof. The ontoness has been proved already (lemma 6.9). So, only the one-to-one-ness has to be checked, in which case one may limit himself to the operator ${ }^{\wedge} \pm$, for the other can be dealt with similarly.

Assume $f \in E(\Delta) \mathbb{H}$ and $\hat{f}_{ \pm}^{*}=0$ as an element of $L_{2}\left(A_{\sqrt{ } \bar{\Delta}}\right)$. One has to show $f=0$. From lemma 6.4 or 6.5 it follows that $\left(V_{ \pm}(\Delta) f\right)^{\wedge}{ }_{0}=0$ in $L_{2}\left(A_{\sqrt{\Delta}}\right)$, and hence $\left(E_{0}(\Delta) V_{ \pm}(\Delta) f\right)^{\wedge}=0$, i.e., $E_{0}(\Delta) V_{ \pm}(\Delta) f=0$. By lemma 6.12 $V_{ \pm}(\Delta) f=0$. But lemmas 6.6 and 6.12 show that $U_{ \pm}(\Delta)\left[V_{ \pm}(\Delta)\right]$ maps $E_{0}(\Delta) \mathbb{H}[E(\Delta) \mathbb{H}]$ one-to-one onto $E(\Delta) \mathbb{H}\left[E_{0}(\Delta) \mathbb{H}\right]$, and $U_{ \pm}(\Delta)$ and $V_{ \pm}(\Delta)$ are inverse to each other. Therefore, $f=0$.

Q.E.D.

The inverses of $\wedge^{\wedge}$ and $\hat{\wedge}$ are now to be determined. This may be accomplished by looking at lemma 6.3. If $f$ is smooth, from lemma 6.3 follows

$$
(E(\Delta) f)(x)=(2 \pi)^{-3 / 2} \int_{A_{\sqrt{\Delta}}} \varphi_{ \pm}(x, \xi) \hat{f}_{ \pm}^{*}(\xi) d \xi
$$

In view of the above equation, which is nothing but an inversion formula, one defines

$$
\check{u}_{ \pm}(x)=(2 \pi)^{-3 / 2} \int_{A_{\sqrt{\Delta}}} \varphi_{ \pm}(x, \xi) u(\xi) d \xi
$$




$$
\check{u}_{ \pm}^{*}(x)=(2 \pi)^{-3 / 2} \int_{A_{\sqrt{\bar{\Delta}}}} \varphi_{ \pm}^{*}(x, \xi) u(\xi) d \xi
$$

The above integrals retain their significance for any $u \in L_{2}\left(A_{\sqrt{\Delta}}\right)$ because of theorem 3.2 and the boundedness of $A_{v^{\prime}}$. It is now clear from the

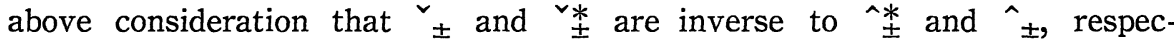
tively. In accordance with the above notation the Fourier inversion will be denoted by ${ }^{\circ}$, which is of course understood to be restricted to $L_{2}\left(A_{\sqrt{4}}\right)$. Further, taking account of lemmas 6.5 and 6.11 one is led to the following lemma. Notice in what follows that by $i d_{E}, i d_{E^{*}}, i d_{E_{0}}$ and $i d_{A}$ are meant the identity operators on the respective Hilbert spaces $E(\Delta) \mathbf{H}, E^{*}(\Delta) \mathbf{H}, E_{0}(\Delta) \mathbf{H}$ and $L_{2}\left(A_{\sqrt{\Delta}}\right)$. (The inner products in $E(\Delta) \mathbf{H}$, $E^{*}(\Delta) \mathbf{H}$ and $E_{0}(\Delta) \mathbf{H}$ are induced by the one in $\mathbf{H}$.)

Lemma 6.14. Let $\Delta$ be as in theorem 5.2. Then the operators ${ }^{2} \pm$ and $\stackrel{\vee}{ } \stackrel{*}{ \pm}$ map $L_{2}\left(A_{\sqrt{\Delta}}\right)$ one-to-one onto $E(\Delta) \mathbf{H}$ and $E^{*}(\Delta) \mathbf{H}$, respectively, and

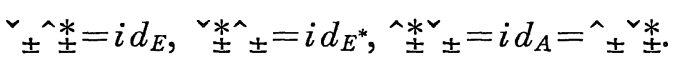

$U_{ \pm}(\Delta)$ maps in a one-to-one manner $E_{0}(\Delta) \mathbf{H}$ onto $E(\Delta) \mathbf{H}, V_{ \pm}(\Delta) E(\Delta) \mathbf{H}$ onto $E_{0}(\Delta) \mathbf{H}, U_{ \pm}(\Delta)^{*} E^{*}(\Delta) \mathbf{H}$ onto $E_{0}(\Delta) \mathbf{H}$, and $V_{ \pm}(\Delta)^{*} E_{0}(\Delta) \mathbf{H}$ onto $E^{*}(\Delta) \mathbf{H}$, and

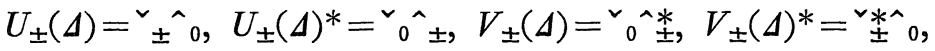

$$
\begin{aligned}
& U_{ \pm}(\Delta) V_{ \pm}(\Delta)=i d_{E}, \quad V_{ \pm}(\Delta)^{*} U_{ \pm}(\Delta)^{*}=i d_{E^{*}} \\
& V_{ \pm}(\Delta) U_{ \pm}(\Delta)=i d_{E_{0}}, \quad U_{ \pm}(\Delta) * V_{ \pm}(\Delta) *=i d_{E_{0}}
\end{aligned}
$$

and thus the pairs $U_{ \pm}(\Delta), V_{ \pm}(\Delta)$ and $U_{ \pm}(\Delta)^{*}, V_{ \pm}(\Delta)^{*}$ are inverse to each other, respectively.

With the above lemma in regard one defines transformation operators $T_{ \pm}$and $T_{ \pm}^{*}$ as follows: Let $u \in L_{2}\left(A_{\sqrt{\Delta}}\right)$ and form $\check{u}_{ \pm} \in E(\Delta) \mathbf{H}$ and $\check{u}_{ \pm}^{*} \in$ $E^{*}(\Delta) \mathbf{H}$. Further, regarding these $\check{u}_{ \pm}$and $\check{u}_{ \pm}^{*}$ as elements of $\mathbf{H}$, form $\left(\check{u}_{ \pm}\right)^{\wedge}=\left(E^{*}(\Delta) \check{u}_{ \pm}\right)^{\wedge} \pm$ and $\left(\check{u}_{ \pm}^{*}\right)^{\wedge} \pm=\left(E(\Delta) \check{u}^{*}\right)^{\wedge}{ }^{*}$ by means of lemma 6.10, which lie in $L_{2}\left(A_{\sqrt{4}}\right)$. Set $T_{ \pm} u=\left(\check{u}_{ \pm}\right)_{ \pm}^{\wedge}$ and $T_{ \pm}^{*} u=\left(\check{u}_{ \pm}^{*}\right)^{\wedge}{ }_{ \pm}^{*}$. Symbolical- 


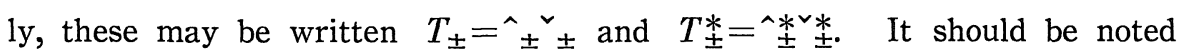
here that $T_{ \pm}^{*}$ is not defined to be the adjoint of $T_{ \pm}$.

Lemma 6.15. Let $\Delta$ be as in theorem 5.2. Then the transformation operators $T_{ \pm}$and $T_{ \pm}^{*}$ defined above are positive-definite, non-degenerate bounded linear operators on $L_{2}\left(A_{\sqrt{4}}\right)$ (and hence they are one-to-one and onto), and

$$
\begin{gathered}
\left(u, T_{ \pm} v\right)_{A_{\sqrt{ }}}=\left(\check{u}_{ \pm}, \check{v}_{ \pm}\right),\left(u, T_{ \pm}^{*} v\right)_{A_{v \bar{\Delta}}}=\left(\check{u}_{ \pm}^{*}, \check{v}_{ \pm}^{*}\right) \quad \text { for } u, v \in L_{2}\left(A_{\sqrt{\Delta}}\right), \\
(f, g)=\left(\hat{f}_{ \pm}^{*}, T_{ \pm} \hat{g}_{ \pm}^{*}\right)_{A_{\sqrt{\Delta}}} \quad \text { for } f, g \in E(\Delta) \mathbf{H}, \\
(f, g)=\left(\hat{f}_{ \pm}, T_{ \pm}^{*} \hat{g}_{ \pm}\right)_{A_{v^{\prime} \bar{\Delta}}} \quad \text { for } f, g \in E^{*}(\Delta) \mathbf{H} .
\end{gathered}
$$

Proof. Let $f, g \in E(\Delta) \mathbf{H}$. Then by lemmas $6.3,6.9$ and 6.10 and the definition of $T_{ \pm}$

$$
\begin{aligned}
(f, g) & =\left(\hat{f}_{ \pm}^{*}, \hat{g}_{ \pm}\right)_{A_{\sqrt{ }}}=\left(\hat{f}_{ \pm}^{*},\left(E^{*}(\Delta) g\right)^{\wedge}\right)_{A_{\sqrt{ }}} \\
& =\left(\hat{f}_{ \pm}^{*},\left(E^{*}(\Delta)\left(\hat{g}_{ \pm}^{*}\right)^{\vee}\right)^{\wedge}\right)_{A_{\sqrt{\Delta}}}=\left(\hat{f}_{ \pm}^{*}, T_{ \pm} \hat{g}_{ \pm}^{*}\right)_{A_{\sqrt{\Delta}}}
\end{aligned}
$$

for any $f, g \in E(\Delta) \mathbf{H}$. Since ${ }^{\wedge} \underset{ \pm}{*}$ is one-to-one and onto from $E(\Delta) \mathbf{H}$ to $L_{2}\left(A_{\sqrt{ }}\right)$ (lemma 6.13), this shows that $T_{ \pm}$is bounded, linear, positivedefinite and non-degenerate, while the last equation but one of the lemma has been already shown. The first equation can be obtained from this by replacing $\hat{f}_{ \pm}^{*}$ and $\hat{g}_{ \pm}^{*}$ by $u$ and $v$ so that $f=\check{u}_{ \pm}$and $g=\breve{v}_{ \pm}$in virtue of lemma 6.14. The assertions concerning $T_{ \pm}^{*}$ may be verified similarly.

Q.E.D.

Fixing an admissible Borel set $\Delta$ as in theorem 5.2, one can define new Hilbert spaces $\mathbf{H}_{ \pm}$and $\mathbf{H}_{ \pm}^{*}$, which are identical with $L_{2}\left(A_{\sqrt{\Delta}}\right)$ as a linear space, by introducing new inner products in $L_{2}\left(A_{\sqrt{\Delta}}\right)$

$$
(u, v)_{ \pm}=\left(u, T_{ \pm} v\right)_{A_{v^{\prime} \bar{\Delta}}} \text { and }(u, v)_{ \pm}^{*}=\left(u, T_{ \pm}^{*} v\right)_{A_{\sqrt{ }}} .
$$

That $\mathbf{H}_{ \pm}$and $\mathbf{H}_{ \pm}^{*}$ equipped with these inner products are Hilbert spaces is clear from the above lemma 6.15 . 
Summarizing and supplementing in an obvious manner what has been discussed so far, one can now state the following theorem.

Theorem 6.16. Let $\Delta$ be a bounded Borel subset of $[0, \infty)$ such that both $\pm \sqrt{\Delta}$ are at a positive distance from $\Sigma$ (or, equivalently, $\Sigma^{*}$ ). Then the following assertions hold. In the following $e, e_{1}$ and $e_{2}$ will denote arbitrary Borel subsets of $\Delta$.

i) There exist absolutely continuous spectral measures $E$ and $E^{*}$ associated with $H$ and $H^{*}$ such that $E(e)$ and $E^{*}(e)$ are (bounded) idempotents, i.e., projections on $\mathbb{H}, E\left(e_{1} \cap e_{2}\right)=E\left(e_{1}\right) E\left(e_{2}\right)$ and $E^{*}\left(e_{1} \cap e_{2}\right)=E^{*}$ $\left(e_{1}\right) E^{*}\left(e_{2}\right)$, and $E(e)\left[E^{*}(e)\right]$ commutes with $H\left[H^{*}\right]$.

ii) There exist one-to-one bounded linear operators $\stackrel{\wedge}{ \pm}^{*}$ and ${ }^{\wedge} \pm$ from Hilbert spaces $E(\Delta) \mathbb{H}$ and $E^{*}(\Delta) \mathbb{H}$ onto $L_{2}\left(A_{\gamma^{\prime}}\right)$ which reduce to

$$
\begin{array}{ll}
\hat{f}_{ \pm}^{*}(\xi)=(2 \pi)^{-3 / 2} \int \overline{\varphi_{ \pm}^{*}(x, \xi)} f(x) d x & \left(\xi \in A_{\sqrt{\Delta}}\right) \\
\hat{g}_{ \pm}(\xi)=(2 \pi)^{-3 / 2} \int \overline{\varphi_{ \pm}(x, \xi)} g(x) d x & \left(\xi \in A_{\gamma^{\prime} \bar{\Delta}}\right)
\end{array}
$$

when $f \in E(\Delta) \mathbb{H}$ and $g \in H^{*}(\Delta) \mathbb{H}$ can be extended to elements of $\mathbb{H}$ as $C_{0}^{\infty}\left(\mathbb{R}^{3}\right)$ functions, where $\varphi_{ \pm}^{*}(x, \xi)$ and $\varphi_{ \pm}(x, \xi)$ are generalized eigenfunctions of $H$ and $H^{*}$ with generalized eigenvalue $\left|\xi^{2}\right| \in \Delta$ introduced in section 3 , such that for any $f, g$ in $E(\Delta) \mathbb{H}$ and in $E^{*}(\Delta) \mathbb{H}$

$$
\begin{gathered}
(E(e) f, g)=\left(\hat{f}_{ \pm}^{*}, \hat{g}_{ \pm}\right)_{A_{\sqrt{e}},} \quad\left(E^{*}(e) g, f\right)=\left(\hat{g}_{ \pm}, \hat{f}_{ \pm \pm}^{*}\right)_{A_{\sqrt{e}}}, \\
(\alpha(H(\Delta)) f, g)=\left(\alpha\left(|\cdot|^{2}\right) \hat{f}_{ \pm}^{*}, \hat{g}_{ \pm}\right)_{A_{\sqrt{\Delta}}},\left(\alpha\left(H^{*}(\Delta)\right) g, f\right)=\left(\alpha\left(|\cdot|^{2}\right) \hat{g}_{ \pm}, \hat{f}_{ \pm}^{*}\right)_{A_{\sqrt{\Delta}}}
\end{gathered}
$$

where $\alpha$ is any bounded Borel function defined on $\Delta, H(\Delta)$ and $H^{*}(\Delta)$ are the restrictions of $H$ and $H^{*}$ to $E(\Delta) H$ and $E^{*}(\Delta) H$, i.e., $H(\Delta)=$ $H E(\Delta), H^{*}(\Delta)=H^{*} E^{*}(\Delta)$, and $\alpha(H(\Delta))$ and $\alpha\left(H^{*}(\Delta)\right)$ are functions of $H(\Delta)$ and $H^{*}(\Delta)$ defined by

$$
\alpha(H(\Delta))=\int_{\Delta} \alpha(\lambda) d E(\lambda), \quad \alpha\left(H^{*}(\Delta)\right)=\int_{\Delta} \alpha(\lambda) d E^{*}(\lambda)
$$

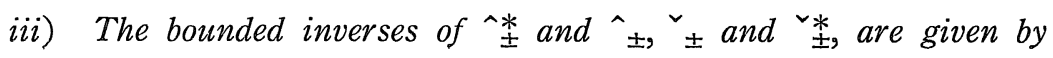




$$
\begin{array}{ll}
\check{u}_{ \pm}(x)=(2 \pi)^{-3 / 2} \int_{A_{v^{\prime} \bar{\Delta}}} \varphi_{ \pm}(x, \xi) u(\xi) d \xi & \left(u \in L_{2}\left(A_{v^{\prime} \bar{\Delta}}\right)\right), \\
\check{v}_{ \pm}^{*}(x)=(2 \pi)^{-3 / 2} \int_{A_{V_{\bar{\Delta}}}} \varphi_{ \pm}^{*}(x, \xi) v(\xi) d \xi \quad\left(v \in L_{2}\left(A_{\sqrt{\bar{\Delta}}}\right)\right) .
\end{array}
$$

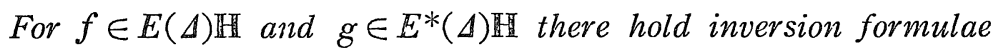

$$
f=\left(\hat{f}_{ \pm}^{*}\right)^{2}, \quad g=\left(\hat{g}_{ \pm}\right)^{2} \stackrel{*}{ \pm}
$$

iv) The operators $\stackrel{\wedge}{ \pm}$ and $\hat{\wedge}_{ \pm}$are unitary when regarded as mapping from $E(\Delta) \mathbb{H}$ and $E^{*}(\Delta) \mathrm{H}$ to $\mathbb{H}_{ \pm}$and $\mathbb{H}_{ \pm}^{*}$, and for any bounded Borel

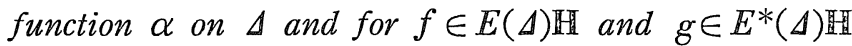

$$
(\alpha(H(\Delta)) f)^{\wedge} \underset{ \pm}{*}=\alpha\left(|\cdot|^{2}\right) \hat{f}_{ \pm}^{*}, \quad\left(\alpha\left(H^{*}(\Delta)\right) g\right)_{ \pm}^{\wedge}=\alpha\left(|\cdot|^{2}\right) \hat{g}_{ \pm}
$$

The inversion formulae stated in iii) are also valid in this case. (Thus the operators $H(\Delta), H^{*}(\Delta)$ and $H_{0}(\Delta)$ are mutually unitarily equivalent, where $H_{0}(\Delta)$ is the restriction of $H_{0}$ to the Hilbert space $E_{0}(\Delta) \mathbb{H}$.)

$v)$ There are operators $U_{ \pm}(\Delta)$ and $V_{ \pm}(\Delta)$ such that $U_{ \pm}(\Delta)\left[V_{ \pm}(\Delta)\right]$ maps $E_{0}(\Delta) \mathbb{H}[E(\Delta) \mathbb{H}]$ one-to-one onto $E(\Delta) \mathbb{H}\left[E_{0}(\Delta) \mathbb{H}\right]$, and $V_{ \pm}(\Delta)^{*}$ $\left[U_{ \pm}(\Delta)^{*}\right]$ maps $\mathbb{E}_{0}(\Delta) \mathbb{H}\left[E^{*}(\Delta) \mathbb{H}\right]$ one-to-one onto $E^{*}(\Delta) \mathbb{H}\left[E_{0}(\Delta) \mathbb{H}\right]$. $U_{ \pm}(\Delta)\left[V_{ \pm}(\Delta)^{*}\right]$ and $V_{ \pm}(\Delta)\left[U_{ \pm}(\Delta)^{*}\right]$ are inverse to each other. These operators are given explicitly by

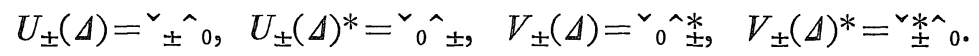

vi) The operators mentioned above in $v$ ) have the intertwining property in the following sense:

$$
U_{ \pm}(\Delta) H_{0}(\Delta)=H(\Delta) V_{ \pm}(\Delta), \quad V_{ \pm}(\Delta)^{*} H_{0}(\Delta)=H^{*}(\Delta) U_{ \pm}(\Delta)^{*}
$$

or, more generally, for any bounded Borel function $\alpha$ on 4

$$
U_{ \pm}(\Delta) \alpha\left(H_{0}(\Delta)\right)=\alpha(H(\Delta)) V_{ \pm}(\Delta), \quad V_{ \pm}(\Delta)^{*} \alpha\left(H_{0}(\Delta)\right)=\alpha\left(H^{*}(\Delta)\right) U_{ \pm}(\Delta)^{*}
$$

(These operators may be called "wave operators" for good reasons.) 


\section{Appendix. Proof of Lemma 2.5.}

Let $\kappa$ be a $Q$-exceptional point. Then there exists a non-trivial $\varphi \in$ H such that

$$
\varphi=Q(\kappa) \varphi=\kappa^{2} A R_{0}\left(\kappa^{2}\right) B \varphi
$$

If $\operatorname{Im} \kappa \geqq 0$, the function

$$
\psi(x)=\frac{1}{4 \pi} \int \frac{\mathrm{e}^{\mathbf{i} \kappa|x-y|}}{|x-y|} B(y) \varphi(y) d y
$$

is a bounded continuous function, and can be seen to satisfy

$$
-\Delta \phi(x)=\kappa^{2} n(x)^{2} \phi(x)
$$

Note that $\psi$ is non-trivial, for the triviality of $\psi$ implies that of $\varphi$.

Now suppose $0 \leqq \arg \kappa \leqq \frac{\pi}{4}$, which implies, if $\kappa=\kappa_{1}+\mathrm{i} \kappa_{2}$,

$$
\kappa_{1}>0 \text { and } 0 \leqq \kappa_{2} \leqq \kappa_{1}
$$

Consider two cases: (1) $\kappa_{2}=0,(2) \kappa_{2}>0$.

Case (1). Multiply (A.1) by $\bar{\phi}$ and its complex conjugate by $\phi$, subtract, and integrate over the ball $|x| \leqq r$, using Green's formula, to obtain

$$
\int_{|x|=r}\left(\frac{\partial \psi}{\partial r} \bar{\psi}-\frac{\overline{\partial \psi}}{\partial r} \psi\right) d S+2 \mathrm{i} \kappa^{2} \int_{|x| \leqq r} \operatorname{Im} n(x)^{2}|\psi|^{2} d x=0
$$

Since the integrand of the first term of (A.3) is rewritten

$$
\left(\frac{\partial \psi}{\partial r}-\mathrm{i} \kappa \psi\right) \bar{\psi}-\left(\frac{\overline{\partial \psi}}{\partial r}+\mathrm{i} \kappa \bar{\psi}\right) \psi-2 \mathrm{i} \kappa|\psi|^{2}
$$

and since $\psi$ is easily seen to satisfy the radiation condition

$$
\frac{\partial \psi}{\partial r}-\mathrm{i} \kappa \psi=o\left(\frac{1}{r}\right), \quad \psi=O\left(\frac{1}{r}\right)
$$

one obtains 


$$
\lim _{r \rightarrow \infty} \int_{|x|=r}|\psi|^{2} d S+\kappa \int \operatorname{Im} n(x)^{2}|\psi|^{2} d x=0
$$

The second term of this equation is never negative, because, by assumption, $\operatorname{Im} n(x)^{2} \geqq 0$. In consequence, the first term must vanish, which in turn implies $\psi=o\left(\frac{1}{r}\right)$. In this case, however, according to a result of Kato $[8], \psi$ must vanish identically outside some ball. Then by the unique continuation theorem one must have $\phi \equiv 0$ on all of $\mathbb{R}^{3}$, a contradiction. So in case (1) $\kappa$ is not $Q$-exceptional.

In case (2), by multiplying (A.1) by $\bar{\phi}$, integrating and taking the imaginary part one is led to

$$
\left(\operatorname{Im} \kappa^{2}\right) \int \operatorname{Re} n(x)^{2}|\psi|^{2} d x+\left(\operatorname{Re} \kappa^{2}\right) \int \operatorname{Im} n(x)^{2}|\psi|^{2} d x=0
$$

(A.2) and the inequality $\kappa_{2}>0$ imply $\operatorname{Im} \kappa^{2}>0$ and $\operatorname{Re} \kappa^{2} \geqq 0$. Therefore, from the above equation

$$
\int \operatorname{Re} n(x)^{2}|\psi|^{2} d x=0
$$

from which one can conclude that $\psi$ vanishes identically outside a ball, since $n(x)-1$ has a compact support by assumption. Arguing as in the preceding paragraph yields $\psi \equiv 0$ on $\mathbb{R}^{3}$, a contradiction.

The first half of the first assertion of the lemma has been proved.

The latter half can be verified in a way similar to the one adopted for case (2) above.

Quite similarly, the assertion involving $\Sigma^{*}$ may be demonstrated.

The last assertion can be proved by looking at (A.4), where the first term vanishes in the present case, and by a unique continuation argument.

\section{References}

[1] Mochizuki, K., Eigenfunction expansions associated with the Schrödinger operator with a complex potential and the scattering theory, Publ. RIMS, Kyoto Univ. 4 (1968), 419-466.

[2] Goldstein, C., Perturbation of non-selfadjoint operators I, II, Arch. Rational Mech. Anal. 37 (1970), 268-296; 42 (1971), 380-402. 
[3] Povzner, A. Ja., On the expansion of arbitrary functions in terms of eigenfunctions of the operator $-\Delta u+c u$, Mat. Sb. 32 (74) (1953), 109-156. (Russian).

[4] Ikebe, T., Eigenfunction expansions associated with Schrödinger operators and their applications to scattering theory, Arch. Rational Mech. Anal. 5 (1960), 1-34.

[5] Shenk, N. A., Eigenfunction expansions and scattering theory for the wave equation in an exterior region, Arch. Rational Mech. Anal. 21 (1966), 120-150.

[6] Avila, G. S. S. and T. P. Haggerty, Spectral representations and scattering for the acoustic equation in an exterior domain, J. Math. Anal. Appl. 27 (1969), 545562.

[7] Kato, T., Perturbation Theory for Linear Operators, Springer, Berlin-HeidelbergNew York, 1966.

[8] Kato, T., Growth properties of solutions of the reduced wave equation with a variable coefficient, Comm. Pure Appl. Math. 12 (1959), 403-425.

[9] Kato, T., Wave operators and similarity for non-selfadjoint operators, Math. Ann. 162 (1966), 258-279.

[10] Ikebe, T., Orthogonality of the eigenfunctions for the exterior problem connected with - 4 , Arch. Rational Mech. Anal. 19 (1965), 71-73.

[11] Saitō, Y., The principle of limiting absorption for the non-selfadjoint Schrödinger operator on $\mathbb{R}^{N}(N \neq 2)$, to appear. 